\title{
Anquilosis mandibular: una frecuente secuela por Noma
}

\section{Mandibular ankylosis: a Noma frequent sequel}

\author{
Fernández García-Guilarte, R.*, Rodríguez Urcelay, P.**, Berenguer Frohner, B.**, \\ González Meli, B.**, Díaz González F.***, Enríquez de Salamanca Celada J.****
}

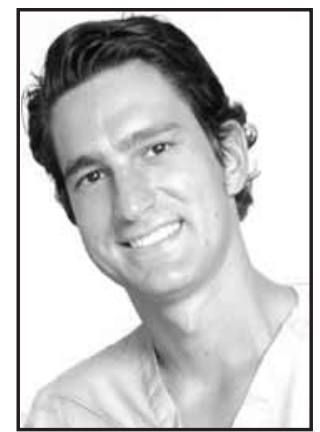

Fernández-García, R.

\section{Resumen}

Conocemos poco sobre el Noma o Enfermedad Cancrum Oris. Esta entidad afecta a niños con malnutrición crónica, poca higiene oral, poco desarrollo sanitario y enfermedades concurrentes debilitantes.

Existe un consenso general sobre que el Noma empieza como una gingivitis; en su fase aguda, puede haber respuesta al tratamiento antibiótico, no obstante, las secuelas tras su curación incluyen daños estéticos y funcionales variables, que pueden requerir cirugía reconstructiva.

Presentamos 3 casos de anquilosis mandibular que en el examen preoperatorio presentaron dimorfismo mandibular, fusión de hueso maxilar y mandibular y anquilosis de la articulación témporomandibular. La ortopantografía y la Tomografía Axial Computerizada (TAC) fueron muy útiles en la planificación de la cirugía. El tratamiento quirúrgico consistió en todos los casos en la resección del hueso anquilótico y la reconstrucción con injerto condrocostal en uno de los casos.

En el postoperatorio se siguió fisioterapia intensiva. Clínicamente todos los pacientes mostraron una gran mejoría en la masticación, la alimentación y el habla, obviamente debido a una buena oclusión.

El problema más común fue la baja colaboración en la rehabilitación mandibular por parte de los pacientes.

\begin{tabular}{|c|c|}
\hline Palabras clave & $\begin{array}{l}\text { Noma, Cancrum Oris, Anquilosis man- } \\
\text { dibular. }\end{array}$ \\
\hline m & 210-161 \\
\hline
\end{tabular}

Little is known about Noma or Cancrum Oris Disease. This entity affects to children with chronic malnutrition, poor oral hygiene, poor environmental sanitation and debilitating concurrent illness.

There is general consensus that noma starts as gingivitis. The acute stage responds readily to antibiotic treatment. The sequelae after healing include variable functional and aesthetic impairments, which require reconstructive surgery.

We report 3 cases of mandibular ankylosis. Preoperative examination revealed mandibular dismorphism, fusion of maxilla and mandible and ankylosis of the temporomandibular joint. Orthopantography and Computed Thomography scan (TCS) were very useful in planning surgery. The surgical treatment performed consisted in ankylotic bone resection in all cases, accompanied with condrocostal graft reconstruction in one case. All patients continued postoperatively with an intensive physiotherapy. Clinically all patients showed an improvement in eating, chewing and speaking, obviously due to good occlusion.

Low collaboration with the mandibular rehabilitation was the most common problem.

\begin{tabular}{|ll|}
\hline Key words & $\begin{array}{l}\text { Noma, Cancrum Oris, Mandibular } \\
\text { ankylosis. }\end{array}$ \\
Numeral Code & $210-161$
\end{tabular}

\footnotetext{
* Médico Interno Residente de Cirugía Plástica, Hospital Universitario La Paz. Madrid. España.

* Cinujano Plástico, Adjunto de la Sección de Cirugía Plástica del Hospital Niño J esús. Madrid. España.

* J Jefe de Senvicio de Cirugía Máxilofacial. Hospital de la Princesa. Madrid. España.

* J Jefe de Sección de Cirugía Plástica del Hospital Niño J esús. Madrid. España

Sección de Cinugía Plástica, Hospital Niño J esús. Madrid. España.
} 


\section{Introducción}

El Noma es en una enfermedad no muy bien descrita en la literatura científica, dado que estos pacientes son remitidos a consulta cuando la enfermedad está en un estadio avanzado. Conocemos poco sobre esta enfermedad en sus estadios iniciales y progresión; tiene un curso clínico rápido y como consecuencia, los pacientes suelen presentar un Noma avanzado cuando son derivados a un hospital (1).

Esta entidad ha sido denominada de diferentes maneras, tales como Noma, Cancrum oris, estomatitis gangrenosa (2), y Cam-tam-na (en Vietnam, significando inflamación oral como un caballo galopante) (3). El término Noma deriva de la palabra griega vо $\mu \eta$, que significa pastar o derivar $(4,5)$.

Afecta a niños con malnutrición crónica, poca higiene oral, mal ambiente sociosanitario, exposición a material fecal animal y humano, exposición a infecciones virales y bacterianas, y debilidad por enfermedades concurrentes, especialmente fiebres eruptivas (4). En el $90 \%$ de los casos, el Noma se desarrolla en menores de 10 años de edad, observándose un pico de incidencia entre 1-4 años, que coincide con el periodo de crecimiento linear retardado en niños necesitados $(1,3,4,5)$. Los infantes son menos susceptibles, dado que la leche por lactación les proporciona proteínas y anticuerpos protectores.

La epidemiología del Noma no ha cambiado mucho a lo largo de los años, excepto que ha habido una reducción en su mortalidad del $90 \%$ al 8-10\%, principalmente debido a los antibióticos modernos $(6,7)$. Entre las enfermedades que comúnmente preceden al Noma se incluyen el sarampión, la malaria, la diarrea severa y la gingivitis ulcerativa necrosante.

\section{CASOS CLINICOS}

Entre los años 200 y 2006, fueron tratados 3 casos de Noma en la Sección de Cirugía Plástica del Hospital Infantil Niño Jesús de Madrid, España. Los 3 individuos procedían de Guinea Ecuatorial y fueron transferidos por una Organización No Gubernamental (ONG) para recibir tratamiento en España. La edad de los niños fue de 7, 12 y 14 años, dos niños y una niña.

Todos los casos referían haber sufrido un absceso facial, probablemente de origen dentario (Fig. 1), y en 1 de los casos, su comportamiento derivó en la formación de una fístula.

Los 3 niños padecían enfermedades concomitantes tales como hipertensión portal debido a cavernomatosis portal, plaquetopenia, parasitosis intestinal, anemia microcítica, etc. el signo más importantes en todos ellos era la asimetría facial, especialmente en el tercio facial inferior (Fig. 2), y la mordida abierta

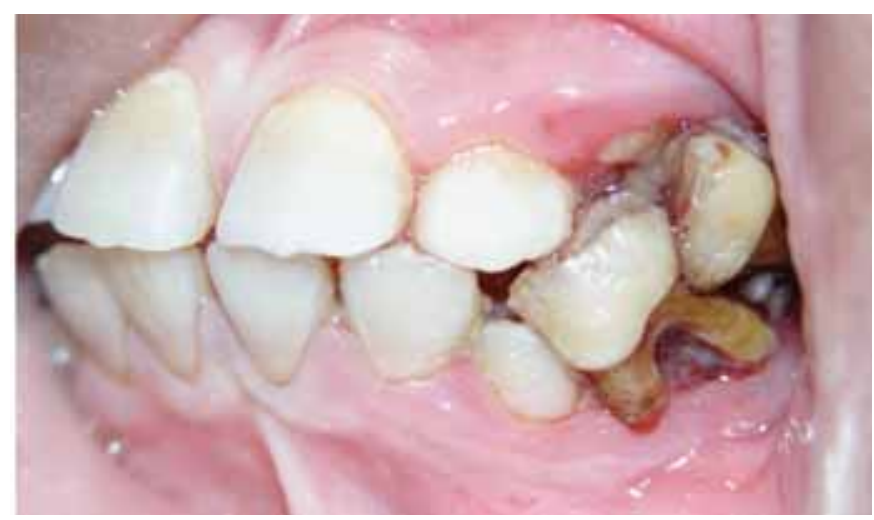

Fig. 1. Gingivitis premolar presente en Caso 1 que evidencia el origen dental de la infección.

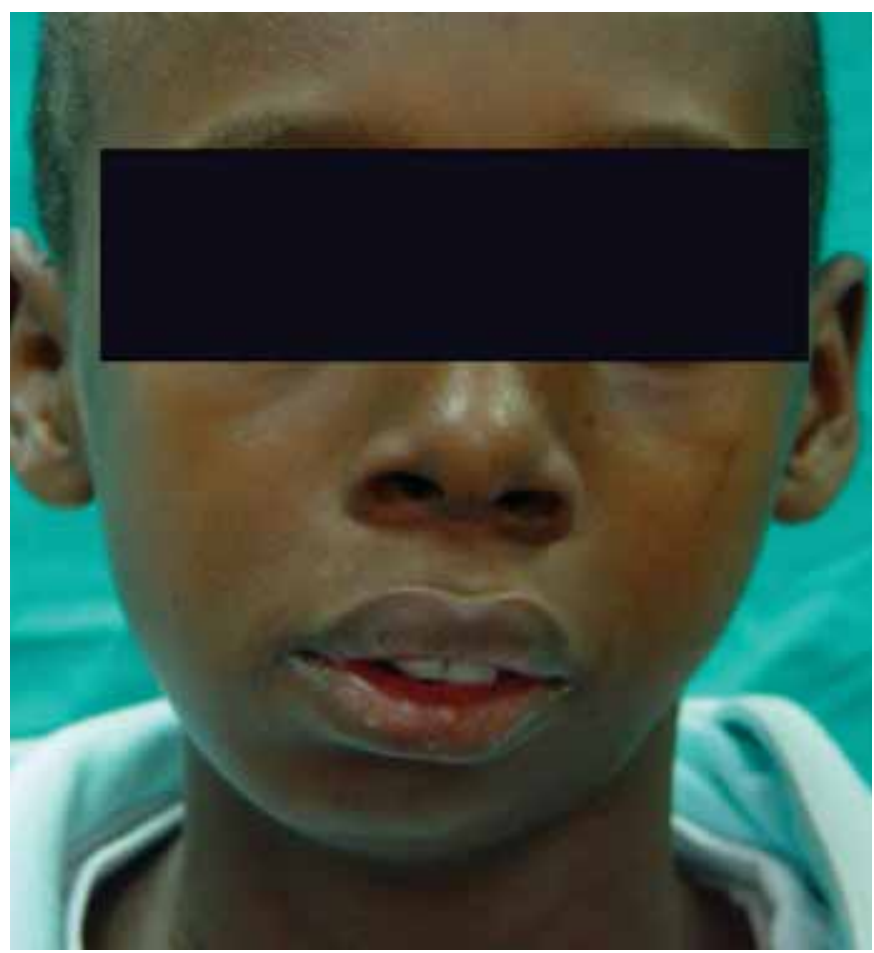

Fig. 2. Caso 3. Asimetría facial, especialmente en el tercio facial inferior.

anterior y lateral con imposibilidad de una apertura bucal mayor.

El exámen clínico reveló dismorfismo mandibular, fusión de la maxila a la mandíbula, osteolisis del ramo-cuerpo mandibular y anquilosis de la articulación mandibular en los 3 pacientes. No presentaban pérdida de tejidos blandos.

La ortopantografía y la reconstrucción tridimensional de imágenes mediante TAC fueron muy útiles para la planificación de la cirugía. Los hallazgos más comunes fueron la desfiguración mandibular con fusión de la pared posterior con la maxila superior y la apófisis pterigoides y ausencia de cavidad articular (Fig. 3).

El tratamiento quirúrgico fue individualizado en función del estadio de esta entidad y del grado de afectación facial; en todos los casos consistió en la resección del hueso anquilótico seguida de reconstrucción con tejido autólogo. Uno de los casos preci- 


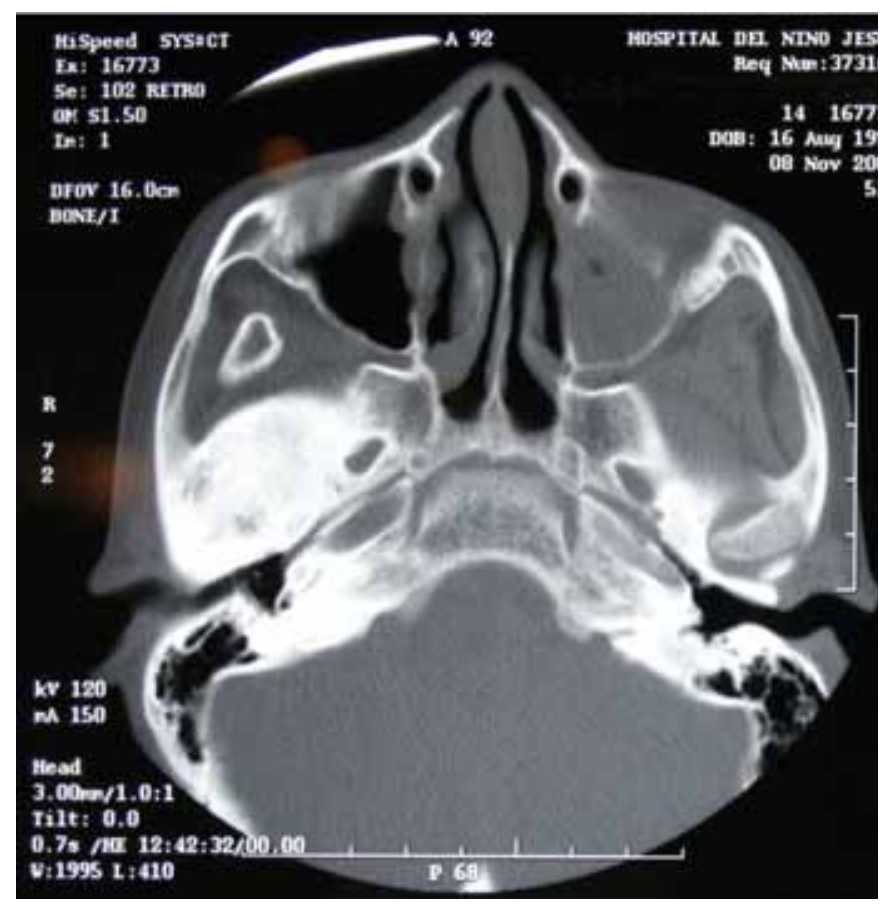

Fig. 3. TAC en Caso 3 que muestra fusión de la pared posterior mandibular con la maxila superior y la apófisis pterigoides, confirmándose un hueso anquilótico con ausencia de cavidad articular.

só reconstrucción mediante un injerto condrocostal una vez extirpado el componente inflamatorio-anquilótico. Otro de los casos requirió un colgajo de mucosa bucal estándar.

Todos los pacientes continuaron postoperatoriamente con fisioterapia intensiva.

Todos los niños toleraron bien la intervención. Hubo un caso de paresia de rama bucal del nervio facial.

Clínicamente todos los casos presentaron una mejoría en la ingesta alimenticia, la masticación y el habla, obviamente debido a una buena oclusión (Fig. 4), que además hacía posible una buena higiene bucal.

La falta de colaboración por parte de los pacientes para la rehabilitación fue el problema más común. A pesar de eso, la mejoría en la apertura bucal fue de 2 - 3,1 cm (Fig. 5). En uno de los pacientes se produjo una ligera recurrencia, pero que no requirió reintervención.

En todos los casos se practicó estudio anatomopatológico de la pieza de resección quirúrgica que determinó la existencia de inflamación aguda y crónica, compatible con osteomielitis biogénica.

\section{Caso 1}

Mujer de 12 años de edad, que refiere haber sufrido absceso facial izquierdo de origen dentario. La clínica predominante en el momento de nuestra valoración fue de imposibilidad para la apertura bucal. El hallazgo más significativo en la TAC y en la Resonancia Magnética Nuclear (RMN) fue la ausencia de glándula parótida izquierda y la deformidad mandibu-

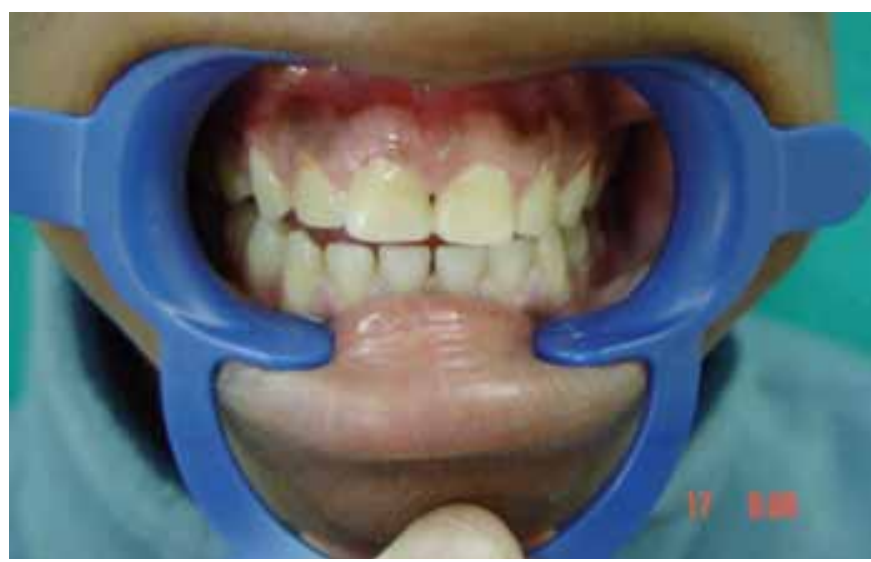

Fig. 4. Caso 3. Buena oclusión tras el drenaje quirúrgico del absceso.

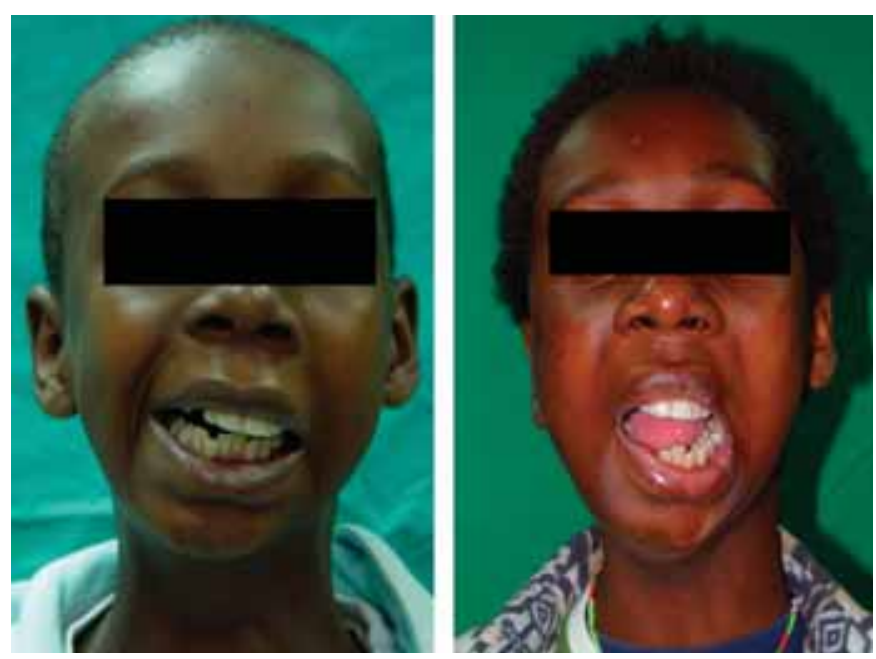

Fig. 5. Caso 3. Mejoría de la apertura bucal tras la resección del hueso anquilótico y reconstrucción con injerto condrocostal.

lar con fusión de sus pared posterior al maxilar superior y apófisis pterigoidea izquierda, con el consiguiente desplazamiento anterior de los cóndilos mandibulares por displasia severa de la articulación témporomandibular.

Dada la situación clínica descrita, se requirió traqueotomía para proceder a la liberación extrarticular de la anquilosis máxilo-mandibular (Fig. 6), extracción de piezas dentarias afectadas y cobertura con colgajo estándar de mucosa. En el postoperatorio inme-

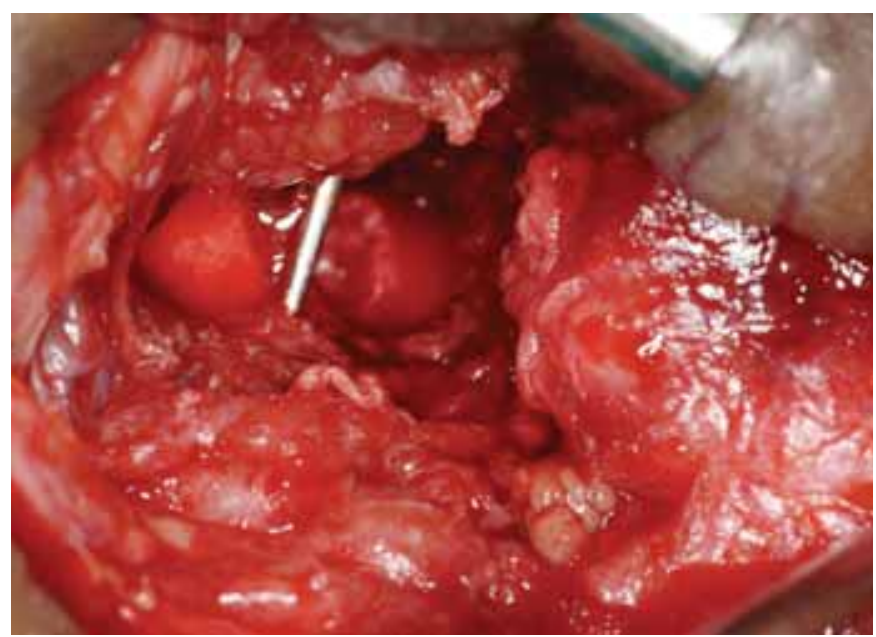

Fig. 6. Caso 1. Liberación extrarticular del hueso anquilótico. 
diato se realizó fijación de la apertura bucal en $2 \mathrm{~cm}$ mediante cuña retromolar.

En el momento actual la paciente mantiene una apertura bucomandibular de $2,4 \mathrm{~cm}$ lo cual permite una alimentación sólida por boca. La confirmación de esta entidad se realizó mediante estudio anatomopatológico que informó de cambios compatibles con osteomielitis crónica.

\section{Caso 2}

Varón de 14 años de edad, que en el momento de la primera consulta presenta una tumoración mandíbulo-témporo-cervical de varios meses de evolución (Fig. 7). Se confirma la presencia de un absceso que se extiende desde región parotídea izquierda por todo el espacio masticador, musculatura pterigoidea ipsilateral y fosa infratemporal (confirmado mediante RMN). Drenamos el absceso y se practica cultivo para anaerobios del material drenado que resulta positivo; se procede a la limpieza del foco osteomielítico piógeno. Mediante estudio anatomopatológico, confirmamos el diagnóstico de enfermedad de Noma dado el componente de inflamación aguda y crónica sin especificidad histológica presente, no evidenciándose granulomas pero sí destrucción ósea del cóndilo temporal.

\section{Caso 3}

Varón de 7 años de edad, que presenta gran asimetría facial por marcada hipoplasia facial derecha. Mediante RMN confirmamos anquílosis mandibular acompañada de gran retracción tisular secundaria y microrretrognatia, lo que permite tan solo una apertura interincisal máxima de $4 \mathrm{~mm}$ además de una maloclusión de clase II.

Se procedió quirúrgicamente a la resección del bloque anquilótico de la articulación témporomandibular derecha mediante osteotomía a nivel del cuello condilar y coronoidectomía derecha, seguidas de reconstrucción mediante injerto condrocostal de interposición. En el momento del alta definitiva, el paciente mantenía una apertura bucal de $3 \mathrm{~cm}$.

\section{Discusión}

La enfermedad conocida como Noma desapareció de los países desarrollados en el siglo XX, excepto por casos asociados a terapia inmunosupresora intensa en pacientes con infecciones por VIH o SIDA, así como en niños nativos americanos con síndrome de inmunodeficiencia combinada severa (8). Sin embargo, el Noma ha permanecido como un importante problema de salud en niños deprimidos en el África subSahariana $(1,9,10)$.

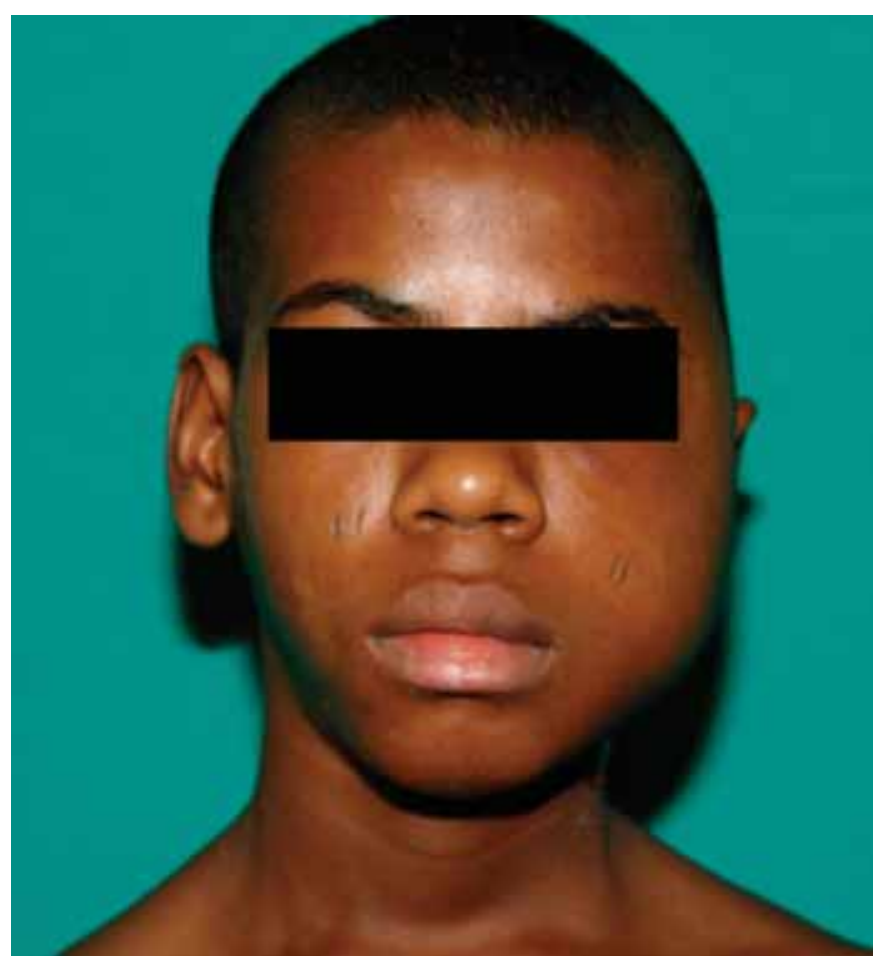

Fig. 7. Caso 2. Edema-tumoración facial consecuente al absceso bucal.

Se trata de una enfermedad infecciosa que empieza como una ulceración gingival que se disemina rápidamente a través de los tejidos orofaciales, estableciéndose un perímetro bien delimitado que rodea un centro negruzco necrótico $(4,11,12)$. La gangrena puede involucrar no sólo a la mandíbula y a la maxila, sino también a la nariz y a los márgenes infraorbitarios. A diferencia de otros procesos infecciosos de la cara, el Noma se disemina a través de barreras anatómicas tales como los músculos (13). Es frecuente también la anemia severa, y la hipoalbuminemia $(10,14)$. Las dificultades en la alimentación debidas a la infección agravan aun más cualquier malnutrición ya existente en este tipo de pacientes.

Es difícil indicar el agente específico principal en la compleja microbiótica del Noma. Se ha especulado con que Borrelia Vincenti y Fusobacterium son las bacterias predominantes en estas lesiones $(15,16)$. También, relaciones simbióticas entre bacilos fusiformes y estreptococos y estafilococos no-hemolíticos (17). Artículos recientes sugieren que además otras bacterias anaerobias estarían también presentes en una alta proporción de lesiones (16). El Fusobacterium necrophorum, productor de dermatotoxinas, podría explicar la rápida progresión de la enfermedad (14).

Existe consenso general acerca de que el Noma empieza como una gingivitis, generalmente en el premolar a molar y región del incisivo mandibular, extendiéndose al surco labiogingival y a la superficie mucosa de la mejilla y al labio $(4,7,10,11)$. Cuando 
la inflamación simultáneamente afecta a la encía y a la superficie mucosa de la mejilla adyacente, la rápida progresión puede finalizar en la perforación de la mejilla $(4,11,13,18)$.

Desde el punto de vista diagnóstico, además de la simple medida de la apertura bucal entre los incisivos centrales superior e inferior con una regla, las ortopantografía puede ser muy útil para determinar el grado de anquilosis.

En la fase aguda de la enfermedad hay respuesta rápida al tratamiento antibiótico. Las secuelas tras la curación incluyen daños funcionales y estéticos variables, que requerirán de posterior cirugía reconstructiva.

Los puntos clave en el manejo durante la fase aguda del Noma son el ingreso hospitalario precoz, la corrección de la deshidratación y de las alteraciones electrolíticas, la rehabilitación nutricional para corregir el déficit energético, las curas diarias de la lesión con gasas humedecidas con antiséptico oral y el tratamiento de las enfermedades sistémicas asociadas ( 7 , 19, 20). Excepto el control de hemorragias secundarias y la retirada de los dientes perdidos, se desaconseja cualquier procedimiento invasivo intraoral. La fisioterapia debe iniciarse durante la fase de curación y continuar tras la cirugía para prevenirse la restricción bucal por cicatrización fibrosa.

La técnica quirúrgica a emplear depende en gran medida de la extensión y de la localización de las lesiones, las facilidades técnicas disponibles, y la competencia del equipo quirúrgico (21), todo en función del defecto tisular existente, de la extensión y de la severidad de la lesión, desde simples colgajos y autoplastias hasta procedimientos complejos que pueden llegar a requerir Microcirugía $(3,4,21)$.

Es muy importante seguir los casos intervenidos y esforzarse por reintegrar a los pacientes a la sociedad, puesto que generalmente se trata de familias analfabetas y sin educación alguna. Desde un punto de vista estético y funcional, el objetivo es hacer que estos pacientes sean respetados miembros de su sociedad, con capacidades de habla inteligibles y con la habilidad de beber, comer y masticar apropiadamente para evitar el aislamiento y la excomunicación. La toma de decisiones debe ser por tanto muy cuidadosa, siendo preferible en algunos casos aplicar una cirugía menos demandante, como podría ser un injerto condrocostal.

Las secuelas del Noma agudo dependen enormemente de la afectación, de la extensión y de la severidad de los tejidos destruidos, así como del estadío de desarrollo del complejo orofacial previo al establecimiento de la lesión. Todo esto puede conllevar el desplazamiento de los dientes, desfiguración, cicatrización intensa, fusión ósea entre la maxila y la mandí- bula, trismus y regurgitación nasal si se pierde la maxila.

Son esenciales los ejercicios continuos postoperatorios para prevenir el trismus y las contracturas.

\section{Conclusiones}

La enfermedad denominada Noma, es una entidad infecciosa bucal en niños que padecen malnutrición crónica, poca higiene oral, mal ambiente sociosanitario, exposición a material fecal animal y humano, exposición a infecciones virales y bacterianas y debilidad por enfermedades concurrentes. Sus signos habituales incluyen dismorfismo mandibular, fusión de la maxila a la mandíbula, osteolisis mandibular y anquilosis de la articulación mandibular.

Para su diagnóstico y plan de tratamiento quirúrgico, las pruebas más útiles son la ortopantografía y la reconstrucción tridimensional de imágenes mediante TAC; la cirugía generalmente consiste en la resección del hueso anquilótico y la reconstrucción mediante tejido autólogo. La rehabilitación postoperatoria es un aspecto fundamental para la obtención de un resultado favorable, pues en la mayoría de los casos se trata de pacientes no colaboradores.

Por todo ello, en los casos de niños, afectados por infección facial, principalmente peribucal, provenientes de un medio socioeconómico no favorable, que cursa con un alto componente inflamatorio facial, asimetría o afectación ósea de distintas características, debemos tener la sospecha de enfermedad Noma. Ante esta circunstancia, lo primordial es distinguir el estadio de la enfermedad y buscar la remisión del proceso no sólo mediante el tratamiento por parte del cirujano plástico, sino de forma interdepartamental dado el importante déficit alimentario, inmunológico, y social que habitualmente subyace en estos pacientes.

\section{Dirección del autor}

Dr. Rubén Fernández García-Guilarte

C/ Pedro Rico, $19-10^{\circ} \mathrm{D}$

28029 Madrid. España.

e-mail: rubenfergar@gmail.com

\section{Bibliografía}

1. Barmes DE, Enwonwu CO, Leclercq MH, Bourgeois D, Falkler WA: "The need for action against oro-facial gangrene (noma)". Trop Med Int Health 1997; 2(12):1111

2. Behanan AG, Auluck A, Pai KL.: "Cancrus oris". British Journal of Oral and Maxillofacial Surgery 2004; 42:267.

3. Marck KW.: "Noma: the face of poverty". Hannover: MIT-Verlag GmbH, 2003: Pp:53-128.

4. Tempest NN.: "Cancrum oris". Br J Surg 1996; 53(1):949. 
5. Enwonwu CO, Phillips RS, Ferrell CD.: "Temporal relationship between the occurrence of fresh noma and the timing of linear growth retardation in Nigerian children". Trop Med Intern Health 2005; 10: 65 .

6. Oji C.: "Cancrum oris: its incidence and treatment in Enugu, Nigeria". Br J Oral Maxillofac Surg 2002; 40(5):406.

7. Marck KW.: "A history of noma, the Face of Poverty". Plast Reconstr Surg 2003; 111(5):1702.

8. Rotbarth HA, Levin MJ, Jones IF, et al.: "Noma in children with severe combined-immunodeficiency" J Pedr 1986; 109: 596.

9. Enwonwu CO.: "Noma: a neglected scourge of children in subSaharan Africa". Bull World Health Organ 1995;73;541.

10. Bourgeois DM, Diallo B, Friech C, Leclerq MH.: "Epidemiology of the incidence of oro-facial noma: a study of cases in Dakar, Senegal 1981-1993". Am J Trop Med Hyg 1999; 61:909.

11. Enwonwu CO.: "Epidemiological and biochemical studies of necrotizing ulcerative gingivitis and noma (cancrum oris) in Nigerian Children". Arch Oral Biol 1972; 17: 1357.

12. Enwonwu CO, Falkler Jr WA, Idigbe EO, Savage KO.: "Noma (cancrum oris): questions and answers". Oral Dis 1999; 5: 144.

13. Evard L, Laroque G, Glineur R, Daelmans P.: "Noma: clinical and evolutive aspect". Acta Stomatol Belg 1996; 93:17.
14. Enwonwu CO, Falkler WA Jr, Idigbe EO, et al.: "Pathogenesis of orofacial gangrene (noma): confounding interactions of malnutrition and infection". Am J Trop Med Hyg 1999; 60: 223.

15. Falker WA, Enwonwu CO, Idigbe EO.: "Isolation of Fusobacterium necrophorum from cancrus oris (noma)". Am J Trop Med Hyg 1999; 60 (1): 150.

16. Falker WA, Enwonwu CO, Idigbe EO.: "Microbial understanding and mysteries of noma (cancrum oris)". Oral Dis 1999; 5(2):150.

17. Berthold P.: "Noma: a forgotten disease". Dent Clin North Am 2003; 47(3):559.

18. Adolph HP, Yuqueros P, Woods JE.: "Noma: a review". Ann Plast Surg 1996; 37:657.

19. Baratti-Mayer D, Pittet B, Montandon D, et al.: "Noma: an "infectious" disease of unknown aetiology". Lancet Infect Dis 2003; 3:419.

20. Campbell DI, Murch SH, Ellia M, et al.: "Chronic T-cell mediated enteropathy in rural West African Children: relationship with nutritional status and small bowel function". Pediatr Res 2003; 54:306.

21. Thiery G, Liard O, Duboscq JC.: "Treatment of noma". Med Trop (Mars) 2002; 62: 193. 